\title{
Opportunities and limitations in the analysis of autistic phenotypes - a conceptual review
}

\section{Éva Mariczáné Csanádi ${ }^{*}$, Ábel Fóthi ${ }^{12} 3$, Péter Pollner ${ }^{4}$, András Lőrincz ${ }^{1}$, Bálint Kovács ${ }^{1}$}

\author{
Affiliation: \\ Pázmány P s 1/a, Budapest, Hungary \\ Budapest, Hungary \\ Budapest, Hungary \\ Budapest, Hungary \\ *Correspondence: \\ Éva Mariczáné Csanádi \\ maricza@inf.elte.hu \\ $+36204304474$ \\ H-1023 Budapest, Frankel Leó street 74-76 \\ Ábel Fóthi \\ fothi.abel@gmail.com \\ Péter Pollner \\ pollner@angel.elte.hu \\ András Lőrincz \\ lorincz@inf.elte.hu \\ Bálint Kovács \\ bolinto@gmail.com
}

${ }^{1}$ Department of Artificial Intelligence, Faculty of Informatics, Eötvös Loránd University, H-1117

${ }^{2}$ Institute of Enzymology, Research Centre for Natural Sciences, H-1117, Magyar Tudósok Körútja 2,

${ }^{3}$ Institute of Archaeogenomics, Research Centre for the Humanities, H-1097, Tóth Kálmán street 4.,

${ }^{4}$ MTA-ELTE Statistical and Biological Physics Research Group, Eötvös Loránd Research Network (ELKH), Dept. of Biological Physics, Eötvös University, H-1117 Pázmány P s 1/a Budapest, Hungary ${ }^{5}$ Health Services Management Training Centre, Semmelweis University, H-1125, Kútvölgyi út 2, 


\section{Conflict of interest statement}

The authors declare a potential conflict of interest and state it below

A.L. has an equity interest in Argus Cognitive Inc

Other authors have no conflict of interest

\section{Author contribution statement}

É.M.Cs: conceptualization, methodology, visualization, writing - original draft; was the main contributor to the conceptualization and framing of the paper, harmonized the report, and provided detailed information about autism-related questions

Á.F., A.L., K.B., P.P.: conceptualization, methodology, writing - review\&editing; contributed to the conceptualization and framing of the paper; provided detailed information about "analysis tools"- related questions; provided critical feedback and helped to improve the paper

All authors read and contributed to the final manuscript.

\section{Acknowledgment - Funding statement}

É.M.Cs., Á.F., K.B. were supported through grant EFOP-3.6.3-VEKOP-16-2017-00002. P.P. received support from the National Research, Development and Innovation Office (no. K128780), EFOP-3.6.3-VEKOP-16-2017-00001 provided by the Hungarian Government and co-financed by the European Social Fund.

A.L. was supported by the project Application Domain-Specific Highly Reliable IT Solutions implemented project (National Research, Development, and Innovation Fund of Hungary, Thematic Excellence Programme no. 2020-4.1.1.-TKP2020) and by the Hungarian Ministry of Innovation and Technology NRDI Office within the framework of the Artificial Intelligence National Laboratory Program.

\section{Ethics statements}

\section{Studies involving animal subjects}

No animal studies are presented in this manuscript.

Studies involving human subjects

No human studies are presented in this manuscript.

\section{Inclusion of identifiable human data}

No potentially identifiable human images or data are presented in this study.

Data availability statement

No datasets were generated or analyzed for this study 


\begin{abstract}
$\underline{\text { Abstract }}$
This paper aims to provide a detailed summary of the opportunities and limitations of determining autistic phenotypes and analyzing the heterogeneity in autistic groups. Our concept is to review the relevant literature and map out the database types, questionnaires, models, and methods used in each research. We examine how (a) the size and nature of the database and (b) the number and type of parameters measured determine what analytic tools should be used and what type of results can be expected. Some review articles are also included to summarize the findings in a broader view. To support our position on the examined aspects, we rely on various articles published on the topic. We discuss the different interpretations and issues in the field of autism questionnaires, models, analytic tools, and subgrouping, focusing on opportunities and limitations.

We emphasize that the individual results in each article can only be interpreted in the light of the methods that brought about the conclusions because the interpretations are influenced by the type of the applied analytical tools. The reviewed literature suggests a heterogeneous palette in autism subtypes instead of distinct, well-characterized subgroups or a singledimensional continuous spectrum.
\end{abstract}

\title{
Keywords
}

heterogeneity, autism models, subgroups in autism, ASD-questionnaires, analysis tools 


\section{$\underline{\text { Introduction }}$}

Autism Spectrum Disorder (ASD) is defined by a range of unusual behaviors of varying severity on a psychosocial level, primarily in communication and collaboration. About 1-2 \% of the population is affected, but the number is increasing (Maenner et al., 2020). The onset of different symptoms is presumably due to genetic and environmental effects. Autism can be characterized by developmental shortcomings in social communication, social interactions that require reciprocity, and cognitive skills that underpin flexible thinking and behavior organization. Because of these multimodal problems, discrepancies are often observed in the behavioral pattern of autistic people in communicating with others, interpreting the reactions of others, and expressing empathy or other feelings. Repetitive movements, word repetitions, attachment to routines, rituals, or extreme interest in a particular topic often appear (American Psychiatric Association \& American Psychiatric Association, 2013).

ASD is a psychosocial disorder that is difficult to diagnose due to its diversified manifestations. To identify the affected individuals as young as possible, make the correct diagnoses, and promote the appropriate development and integration, it is essential to develop accurate, scientifically demanding diagnostic and evidence collecting systems and interpret the gathered data correctly.

Since both autism traits and the parameters of the questionnaires used in the research in this area are rather heterogeneous, it is challenging to identify subgroups of ASD individuals. One may try to use symptoms, for example, for the purpose of treatment indications or finding the underlying causes of subtypes for clustering ASD people. Sometimes clustering is hindered by differences in the labeling of the designated symptoms.

Heterogeneity in the ASD population can be analyzed with dimensional- (spectrum-, networkor palette-) models or with subtype-models. We found several papers that use one of these 
models. However, these contradictory models seem to differ only by the aspects of describing the same phenomenon and depend on the study's goal. We think both the concept of the spectrum (i.e., continuity) and the palette concept (i.e., clustering, possibly with overlaps) have relevance. Their respective supports depend on the specific goal of the individual studies.

Our review paper focuses on the different aspects of autism phenotypic subtyping research regarding the data, questionnaires, methods, and models. We integrate our findings using papers and review articles of this area. We summarize the different variations, possibilities, and methodic limitations of evidence collecting and evaluation methods.

\section{Methods of paper selection}

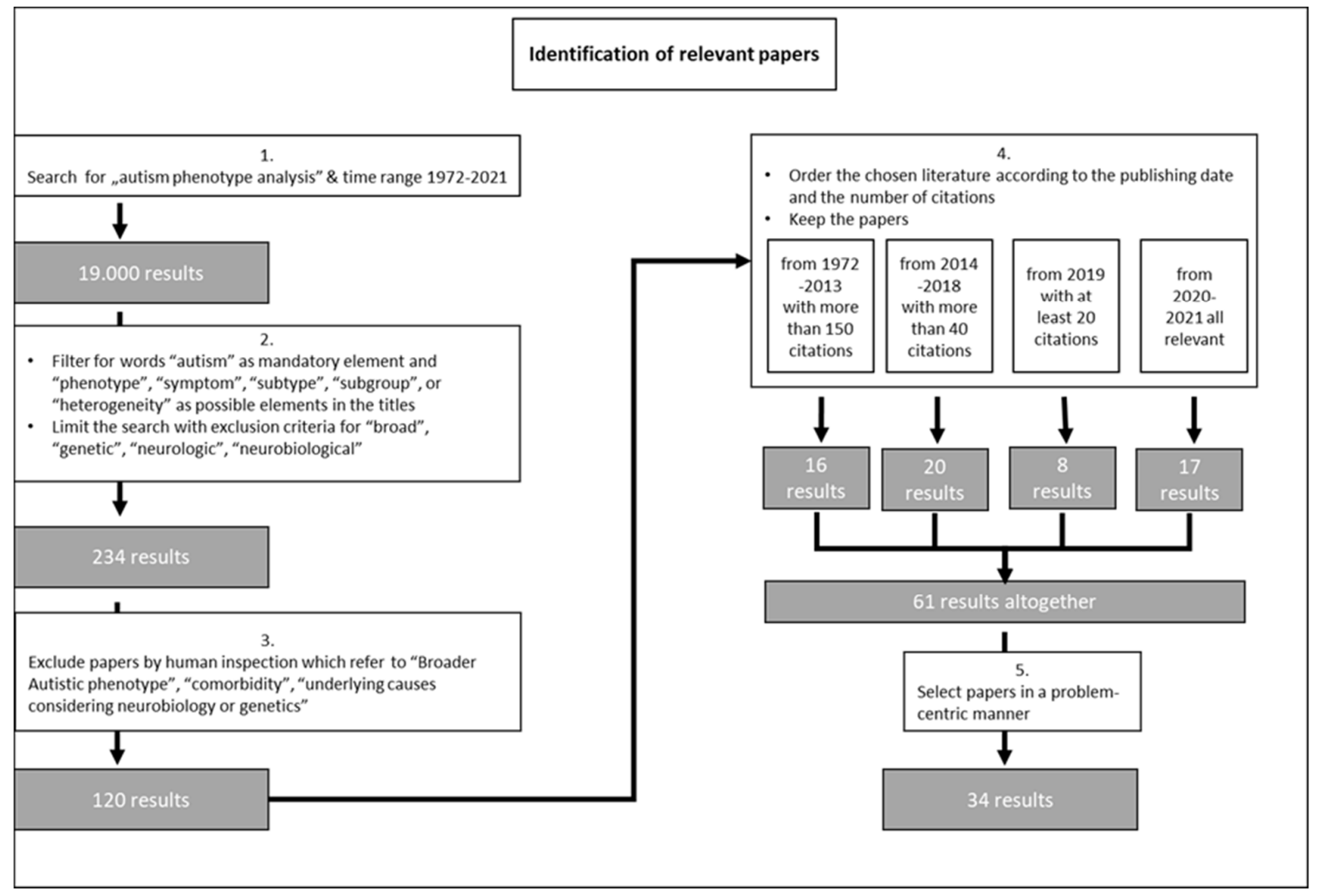

\section{Figure 1}

Identification of relevant research and review papers: This flowchart shows the process with numbered decision-steps and the resulting number of selected papers after each step 
We conducted our review using the considerations and guidelines of "PRISMA" (Page et al., 2021) and „Grade-CerQual” (What Is the GRADE-CERQual Approach, 2019) for examining the papers chosen. We gathered the literature via Google Scholar until 2021 April. We first searched the literature for "autism phenotype analysis" between 1972-2021. Since this search brought more than 19,000 results, we narrowed our search with filtering for the words "autism" as a mandatory element and "phenotype" "symptom," "subtype," "subgroup," or "heterogeneity" as possible elements in the titles of the papers. We limited the search with exclusion criteria for "broad," "genetic," "neurologic," "neurobiological.” We obtained 234 documents with this method. By human inspection, we have excluded papers that refer to "Broader Autistic phenotype," "comorbidity," "underlying causes considering neurobiology or genetics" that were not excluded beforehand. These are outside of the scope of our review, although it would be essential to expand the work in these directions, too. We obtained 120 papers. Finally, we ordered the chosen literature according to the publishing date and the number of citations. We kept the documents from 1972-2013 with more than 150 citations (16 articles), the papers from 2014-2018 with more than 40 citations (20 pieces), from 2019 with at least 20 citations ( 8 articles), and all from 2020-2021 (17 articles), which resulted in 61 articles. A further selection of articles was executed in a problem-centric manner and for the sake of eliminating overlaps. This selection resulted in a subcollection of 34 papers. This way, we have narrowed our collection to 8 reviews and 26 research papers to have a broad collection of examples on analyzing autistic phenotypes.

We regrouped these papers according to the following types: reviews, research-based general analyses, control-case studies, dimensional model - studies, and subtype model - studies. The selected subcollection of 34 papers was analyzed regarding the database type and size, model type, questionnaires and methods used, results, and subgroups (if any) found in the chosen 
paper. We also examined the methodological possibilities and limitations that might emerge from coherence and adequacy problems concerning sample size and representativity or data collection with different questionnaires. Based on the detailed results of the selected papers, we present examples that support our claims, namely that research on autism heterogeneity should be designed with the specificities of databases, questionnaires, and methods to be used kept in mind, and that the possible subgroups that are found, might depend on several parameters from the above.

\section{$\underline{\text { Results - Statistics of the selected papers }}$}

Details of our findings can be found in Supplementary I.A.1-2, "results from chosen articles" excel table.

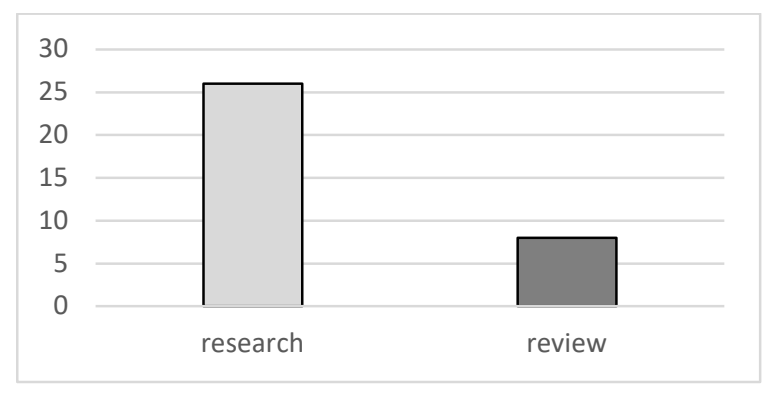

Figure 1

Type of papers: Our subcollection of selected papers consists of 26 research papers and eight reviews

We had 26 research studies and eight review papers to examine as described above. (Figure 2)

\section{Model types}

We found 10 case-control studies and 26 works using heterogeneity models, from which 7 pieces were constructing dimensional models and 17 studies with subtype-based results. 6 case-control studies also subtyped the autistic group (Figure 3). 


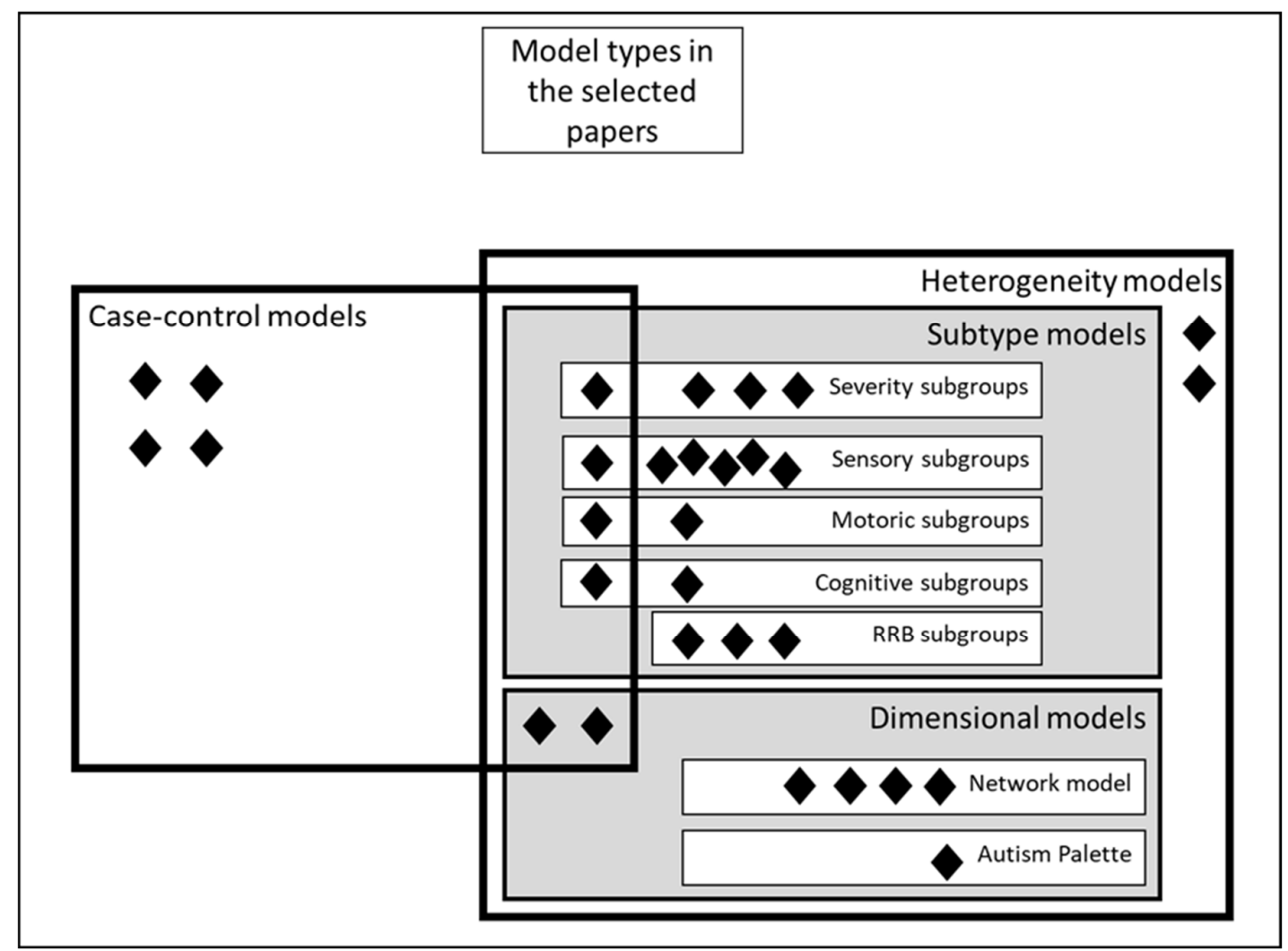

Figure 3

Occurrence of the different model types in the examined studies

Four case-control studies, six studies with both case-control model and heterogeneity model, twenty studies with varying kinds of heterogeneity models (RRB: restricted and repetitive behaviors)

\section{Databases}

As for the databases, two types appeared in the examined works. In case-control studies, the databases consist of two samples: typically developing (TD) individuals and autistic (ASD) individuals the other sample. The differences between the two groups are examined during the study. In heterogeneity studies (such as subtype-based studies or those using dimensional models), the database consists of a single sample of autistic individuals, and the composition of this group is analyzed. 


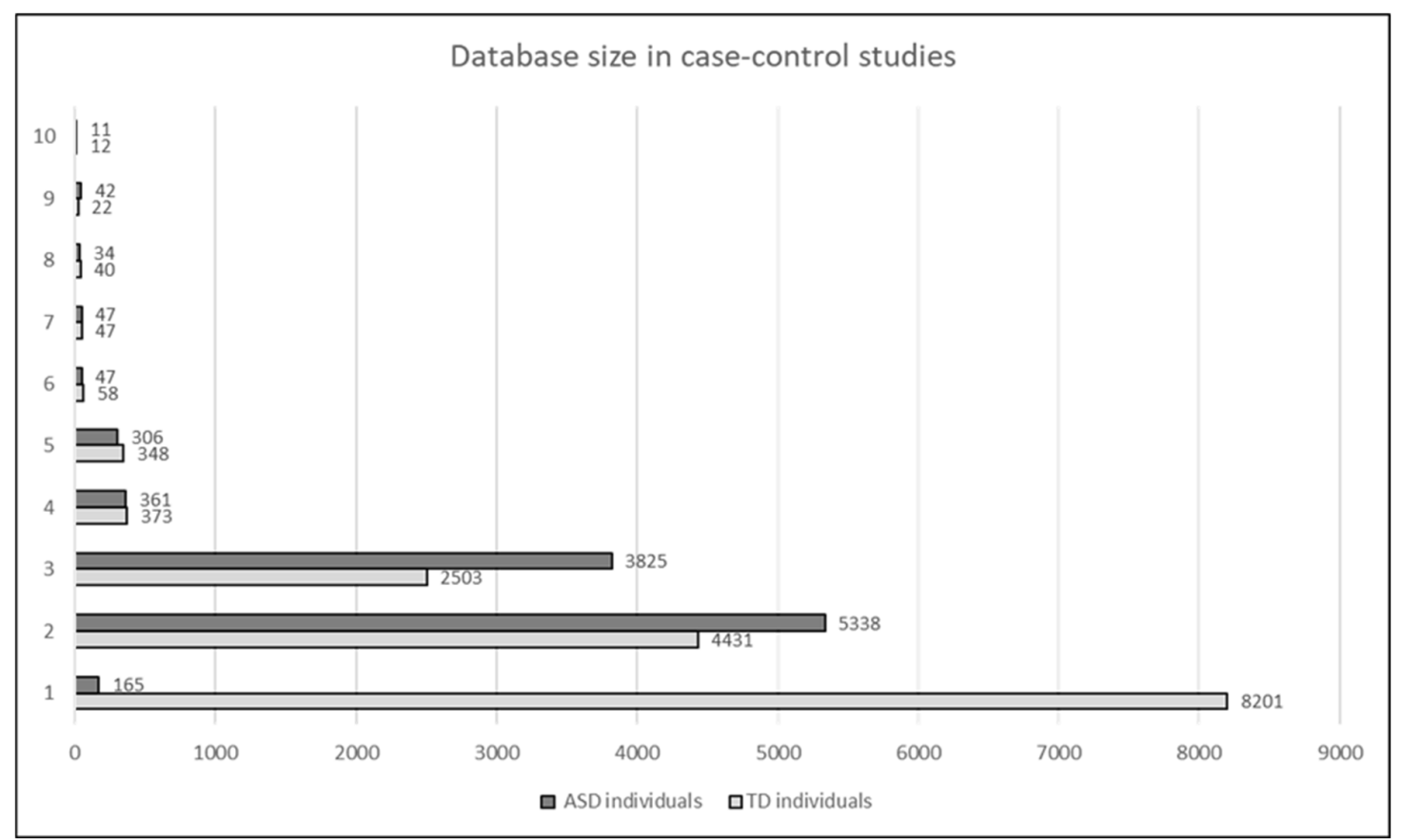

Figure 2

Size of database in case-control studies

Database size varied in the examined works from 12 to 8201 individuals for the TD group and 11 to 5338 individuals for the autistic group

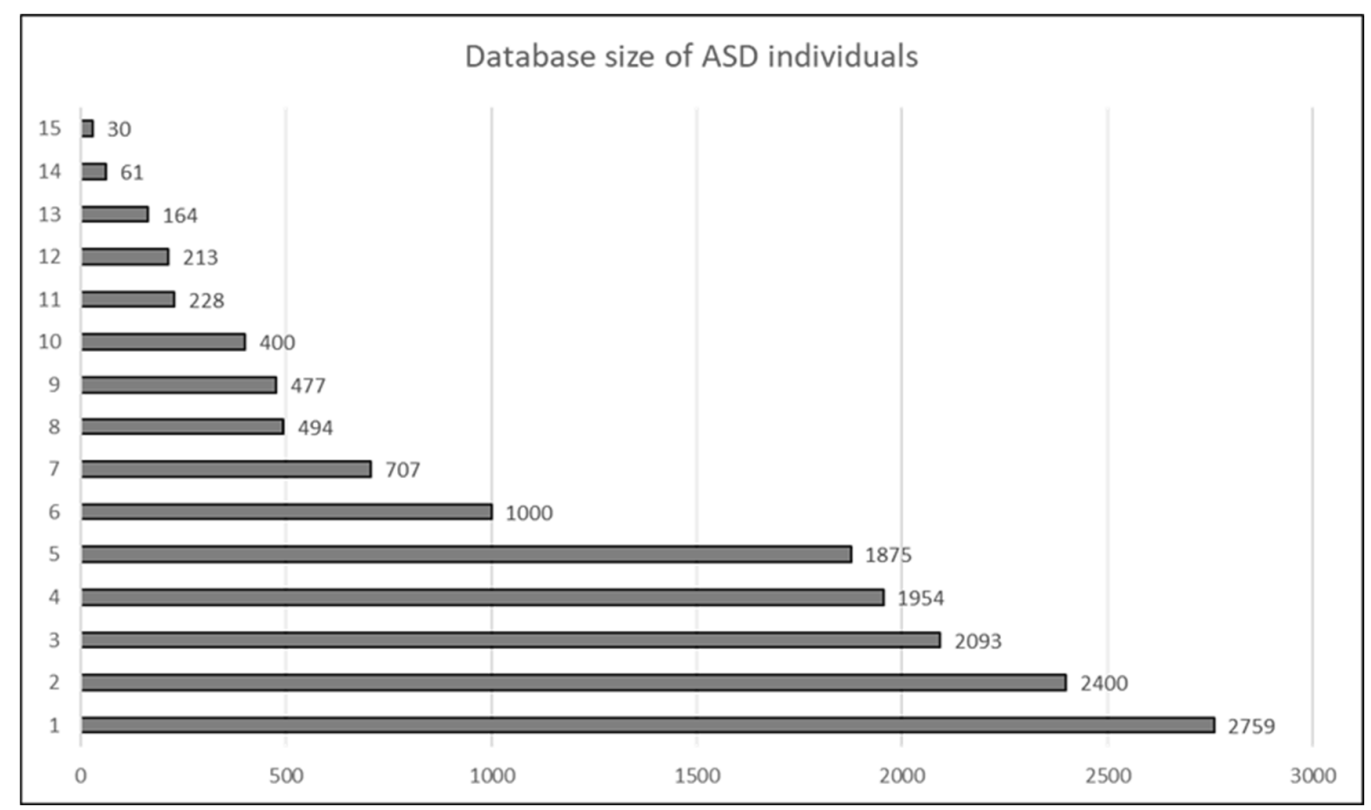

Figure 3

Database size as the number of ASD individuals in heterogeneity studies

Database size varied from 30 to 2759 individuals in the examined works 
The size of the databases varied greatly from research to research, from 12 to 8201 individuals for the TD group and 11 to 5338 individuals for the autistic group in case-control studies (Figure 4) and from 30 to 2759 autistic individuals in heterogeneity studies (Figure 5). The use of smaller databases may limit the conclusions drawn from such studies.

\section{Questionnaires}

Twenty-one studies mentioned using 17 different ASD questionnaires, other behavioral tests, and IQ- or cognitive tests from the examined research papers. The number of questionnaires used in each study ranged from 1 to 8 . (Figure 6)

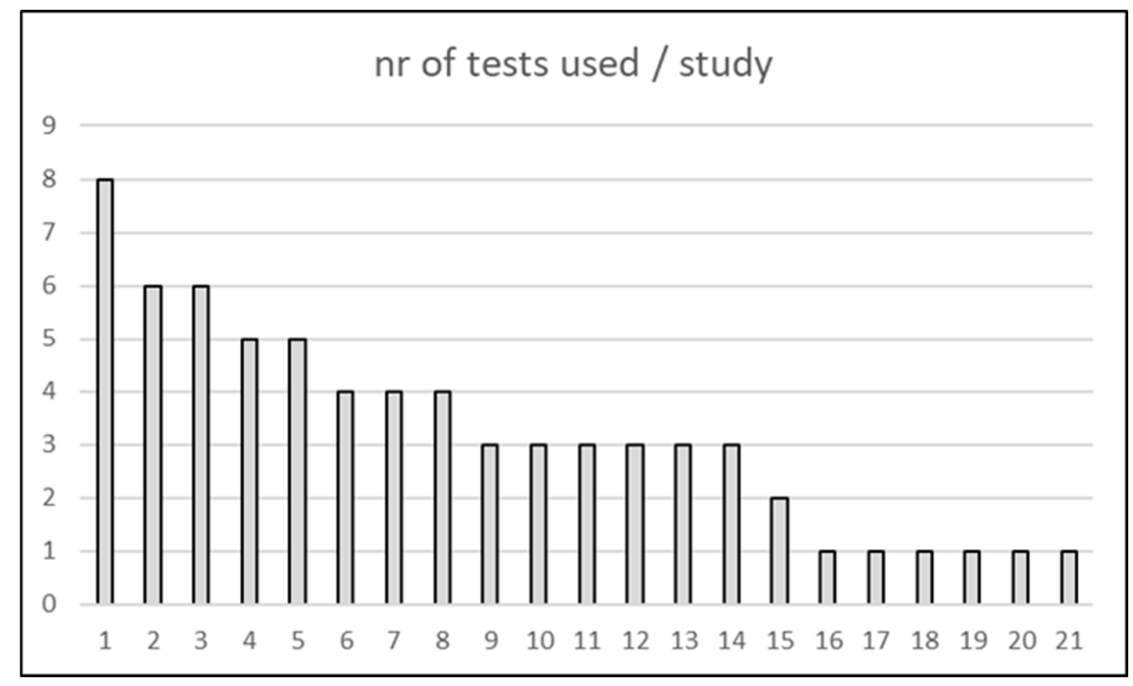

Figure 4

Occurrence of questionnaires used in each examined study

The number of used questionnaires ranged from 1 to 8 in the reviewed studies 


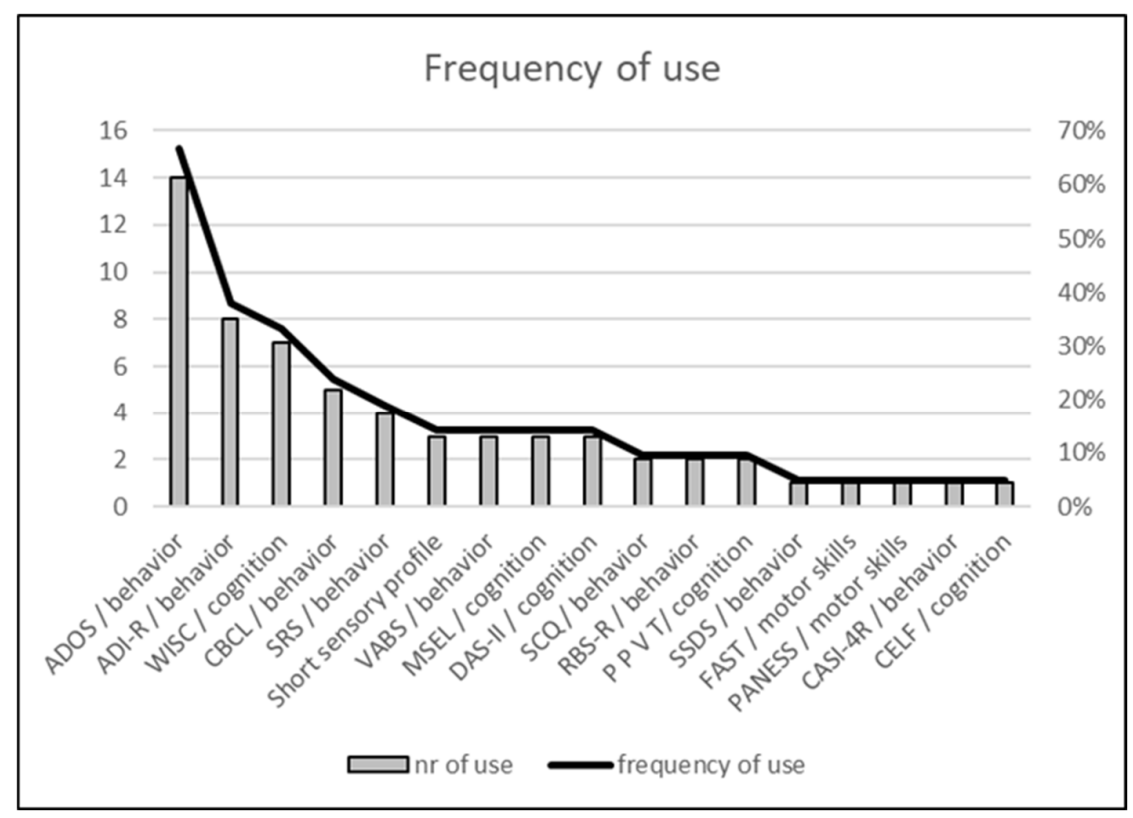

\section{Figure 5}

Frequency of use of the different tests

The most frequently used tests were ADOS, ADI-R, and WISC, used in $66 \%, 38 \%$, and $33 \%$ of the examined papers, respectively ( ADI-R: Autism Diagnostic Interview-Revised, ADOS: Autism Diagnostic Observation Scheme, CASI-4R: Child and Adolescent Symptom Inventory-4R, CBCL: Child Behavior Checklist, CELF: Clinical Evaluation of Language, DAS II: Differential Ability Scales, Second Edition, FAST: Functional Analysis Screening Tool, MSEL: Mullen Scales of Early Learning, PANESS: Physical and Neurological Examination for Soft Signs, PPVT: Peabody Picture Vocabulary Test, RBS-R: Repetitive Behavior Scale-Revised, SCQ: Social Communication Questionnaire, SRS: Social Responsiveness Scale, SSDS: Stanford Social Dimensions Scale, VABS II: Vineland Adaptive Behavior Scale-II, WISC: Wechsler Intelligence Scale for Children)

The most frequently used tests (Figure 7) are ADOS (used 14 times/21 studies), ADI-R (used eight times/21 studies), CBCL (used five times/21 studies), and SRS (used four times/21 studies) for measuring behavioral or autistic traits, and WISC (used seven times/21 studies) for measuring IQ and cognitive abilities. Other mentioned tests are used in only 1-3 studies each. 


\section{Analysis tools}

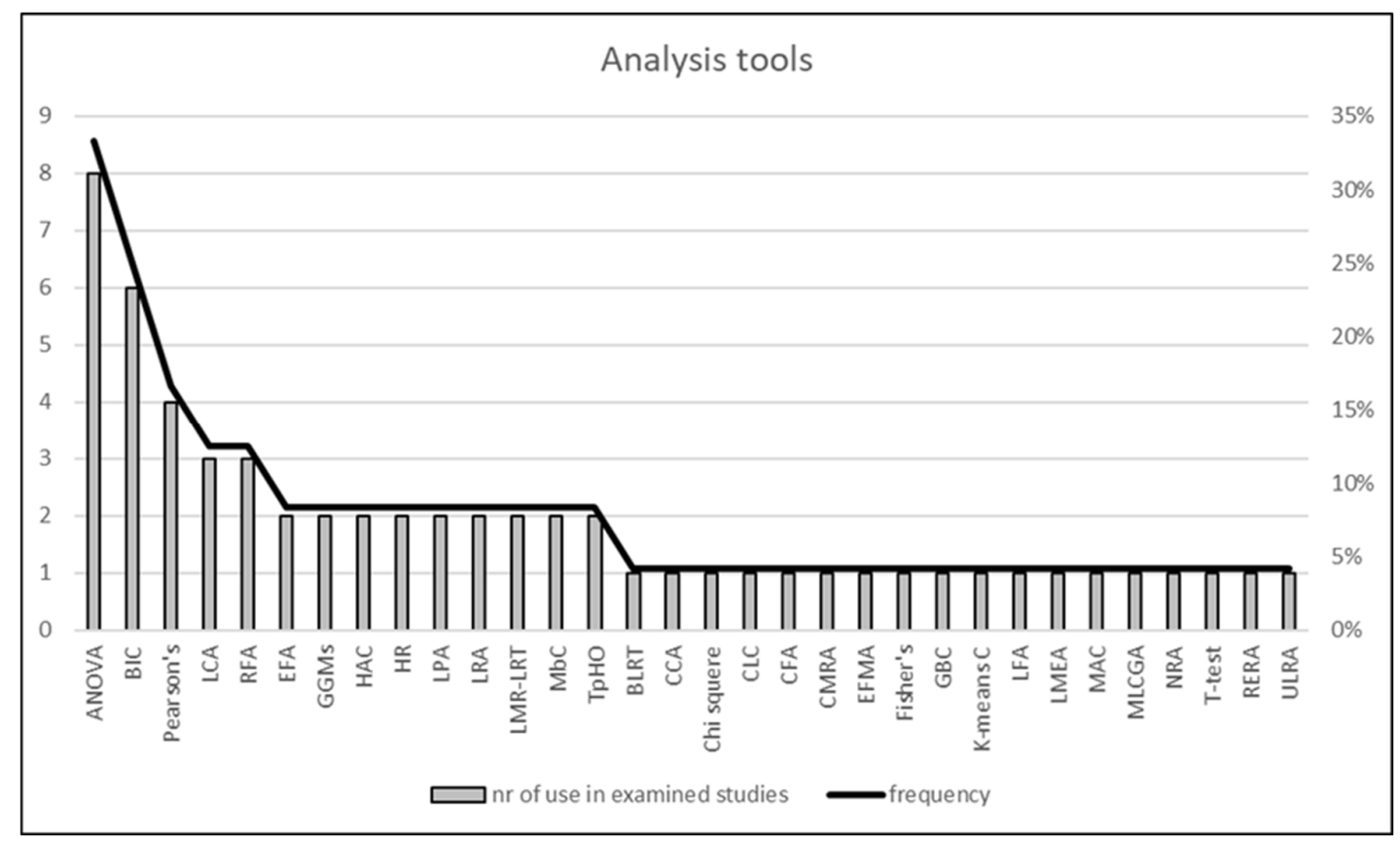

Figure 6

Mentioned analysis tools in examined studies

The most frequently used tests are ANOVA (used in 30\% of the examined studies), BIC (used in 23\% of the reviewed studies), Pearson's correlation (used in 15\% of the analyzed studies), Latent Class Analysis (used in 11\% of the examined studies) and Random Forest (used in $11 \%$ of the reviewed studies). Other tools are used in only 1-2 studies each.

analysis tools mentioned: analysis of variance: ANOVA, Bayesian Information Criterion: BIC, Pearson's correlations: Pearson's, Latent class analysis: LCA, Random Forest Algorithm: RFA, Exploratory factor analysis: EFA, Gaussian Graphical Models: GGMs, Hierarchical agglomerative clustering: $H A C$, Hierarchical regression: HR, Latent profile analysis: LPA, Linear Regression Analyses: LRA, Lo-Mende-Rubin likelihood ratio test: LMR-LRT, Model-based cluster analysis: MbC, Tukey post-hoc comparison: TpHO, BLRT: bootstrap likelihood ratio test: BLRT, Canonical correlation analysis: CCA, Chi squere, Complete linkage cluster analysis: $C L C$, Confirmatory factor analyses: $C F A$, Correlation \&multiple regression analyses: CMRA, Exploratory factor mixture analysis: EFMA, Fishers ExactTest: $\quad$ Fisher's, Gradient Boosting classifier: $G B C, K$-means cluster analysis: $K$ means $C$, Latent factor analysis: LFA, Linear mixed-effects analysis: LMEA, Multivariate analysis of covariance: MAC, Multivariate latent class growth analyses: MLCGA, Neighborhood regression approach: NRA, One sided t-test: T-test, Random effects regression analysis: RERA, Unadjusted linear regression analyses: ULRA

Detailed information on these analysis tools can be found in Supplemental I.B. - "methods in papers" excel file 
The usage of different analysis tools (Figure 8) in the examined papers is as heterogeneous as the autism palette itself. The reviewed papers mentioned 33 different statistical methods in the 24 studies that listed the analysis tools used in their research. The most frequently used tests are ANOVA (used eight times/24 studies), BIC (used six times/24 studies), Pearson's correlation (used four times/24 studies), Latent Class Analysis (used three times/24 studies), and Random Forest (used three times/24 studies). Other tools are used in only 1-2 studies each.

The results obtained from the chosen studies vary considerably. We hypothesize that discrepancies originate because the researchers used databases of different sizes, chose different parameters from different questionnaires, and analyzed them with different statistical tools.

\section{Discussion - Aspects of analyzing autistic phenotypes}

When examining the aspects of analyzing autistic phenotypes, we found several different approaches in the chosen papers. Many opportunities and limitations emerge when analyzing the traits of autistic individuals, as several aspects need to be considered. Model types, data usage, pre-selected aspects, the chosen questionnaires, and methods used all affect the results that can be obtained. Here, we outline the aspects of autism phenotype research by synthesizing the information collected from the chosen articles. 


\section{Model types, database types, and data usage in analyzing autistic phenotypes}

When designing research on the heterogeneity of autism, many aspects need to be considered. The selection of the appropriate database, the necessary questionnaires, and the methods used for the analysis depend on the nature and purpose of the study.

\section{Models}

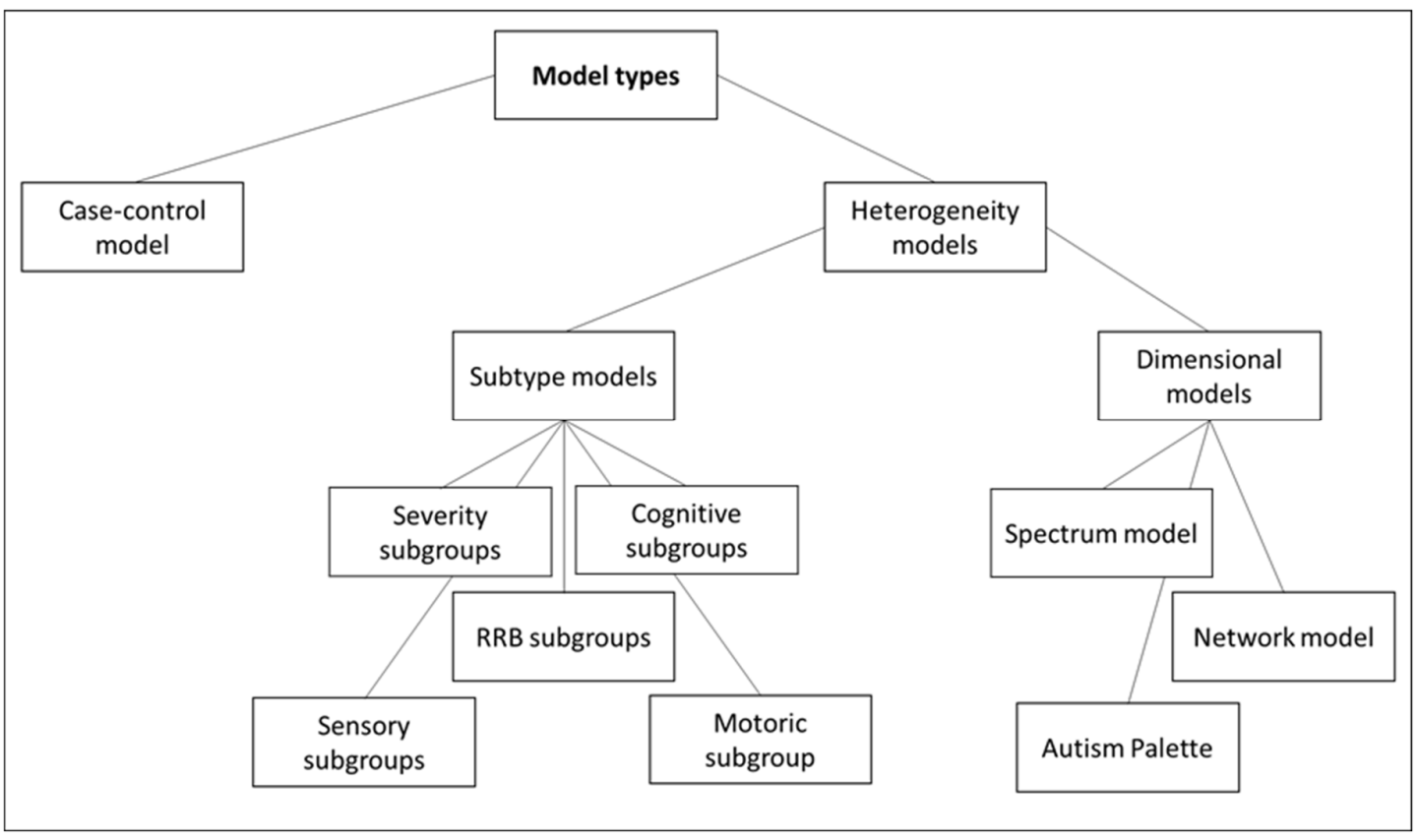

Figure 7

Model types and possible subgroups

Based on the purpose of the study, one can use different models to examine the chosen database

In terms of model types, one can distinguish between case-control models and heterogeneity models (Figure 9). 
When conducting case-control studies, studies identify ASD individuals in the whole population (Randall et al., 2016) or choose two samples of known TD- and ASD individuals. The purpose of a case-control study is to find differences between ASD and TD individuals, usually based on previously chosen characteristics (Dziuk et al., 2007) (Norcia et al., 2021). Some researchers assume that at least the TD group is homogeneous in these studies. Sometimes, they also consider the ASD group as homogeneous concerning the chosen aspect of their research (Macari et al., 2021). In reality, some characteristics of TD individuals and ASD subgroups might overlap (Kim et al., 2018), different causes might lead to the same symptoms (Constantino \& Charman, 2016), and the combination of ASD symptoms that occur in one individual may be caused by several different mechanisms (Katuwal et al., 2016).

Furthermore, the group of typically developing individuals can also be heterogeneous (Becht et al., 2016), and differences within the autistic group might be caused by the original heterogeneity of the population (Feczko et al., 2018) (Feczko et al., 2019). Therefore, a wide variety of details need to be considered when designing research examining the heterogeneity in autism. In particular, averages over typically developing individuals may diminish the power of comparisons and predictions.

Heterogeneity models identify subtypes or dimensions in a group of ASD individuals, usually from a previously given aspect (see subsection „,Aspects in the formation of subgroups" for details). Wing first mentioned the possible existence of the heterogeneous spectrum of symptoms in autism in 1975 (WING, 1996). From that early date to the present, countless research has been conducted to analyze this phenomenon. The most used heterogeneity models are the subtype- and the dimensional models.

Subtype or stratified models divide the sample into groups based on predefined values of one or more aspects. Groups can be formed according to a previously designated aspect 
(supervised approach) or through clustering data beforehand and forming groups afterward (unsupervised method). Several studies use subtype models in their analysis (Wiggins et al., 2017) (Ankenman et al., 2014) (Russell et al., 2019). It could be beneficial to use subtype (stratified) models if the goal is predicting treatment responses. On the other hand, dimensional models might have advantages when seeking underlying biological mechanisms (Lombardo et al., 2019) (Kim et al., 2018). According to the first type of dimensional models, the spectrum model, the values of each examined characteristic are continuous variables. The continuous nature of descriptive variables expresses the assumption that individuals cannot be divided into clear categories. The use of spectrum models is more common in works that examine the causes of symptoms and not the phenotypic aspects (Hollander et al., 1998) (Tang et al., 2020).

Network models are another type of dimensional models that displays several intertwined characteristics of interacting factors (Anderson et al., 2015). These models are common in studies that use data-clustering methods (Baadel et al., 2020) (Haslbeck \& Fried, 2017).

Autism palette model (Fóthi et al., 2020) is a reasonably new, synthesizing approach among the studies analyzing the heterogeneity of autism. The authors of this model hypothesize that the leading causes of autism are minor neurobiological dysfunctions. These could occur at any point in the neural network concerning perception, processing, and response associated with social contact. These dysfunctions or impairments would not cause any problem if there were only a few of them. Still, when combined, they can hinder the individual in connecting with others due to the specificity of autism, the task of communication tasks that can be spoiled in diverse ways and diverse degrees. The authors call these impairments "the basic colors of autism, whereas the combinations of diverse impairments make the palette of autism" (Fóthi et al., 2020). According to this point of view, autism heterogeneity is caused by the 
combination of several dysfunctions, making a dissimilar palette of causes, which concludes in a different symptom-palette in each individual.

\section{Data}

Studying an area showing such heterogeneous data as autism, the research must meet specific statistical criteria. Concerning the database, the most important features are the type, size, and representativity. The type of the database depends on the type of the research model. For casecontrol studies, databases contain two distinct samples of TD and ASD individuals; there is only one sample of ASD individuals for heterogeneity studies. As for the size of the database, it depends on the study's goal. Examining specific subgroups means analyzing only a few dimensions; in that case, a smaller sample size could be appropriate (Feczko et al., 2019). In contrast, a more extensive database is needed to examine multiple dimensions of the complete sample. It is more likely to achieve high statistical power if the selection is large and representative. A too-small sample size might cause a minor or inflating effect size, while moderate-sized samples can work if effect size (statistical power) is appropriate (Anderson et al., 2015). The use of "big data" is highly recommended, and the used database should also be broad. That means not only a large number of involved individuals but a comprehensive set of individual data from different behavioral tests and a medical background can be significant (Kim et al., 2018) (Lombardo et al., 2019).

Several big databases gather relevant data from autistic individuals and their family members. They usually collect genetic samples in addition to medical and behavioral data to provide a better understanding of the underlying causes of phenotypic traits in autism. Here we list some examples of the most extensive databases on autism in the U.S. 
AGRE (Autism Genetic Resource Exchange) is a "collection of genetic, medical, and behavioral data from over 1700 well-characterized multiplex and simplex families" (AGRE Program Description, n.d.). Simplex families have one child affected with ASD, with unaffected parents and siblings, multiplex families have more than one ASD child. Data collection started in 1997 and is still going on. This database collects information through 15 phenotypic and medical questionnaires, supplementary demographic data, and collects genetic data of 7 different aspects. The list of tests is in Supplementary II.A.

MSSNG also has an impressive database with more than 5000 cases, uses eight behavioral questionnaires: ADOS, ADI-R, VABS, RBS-R, SRS, SCQ, CBCL, and ABC (MSSNG, n.d.) (C Yuen et al., 2017)

Other databases are at Simon's Foundation, SFARI (Simons Foundation Autism Research Initiative). These databases also gather medical, behavioral, and genetic information about the persons contributing (SFARI | SPARK Phenotypic Measures, n.d.) (SFARI | SPARK Demographic and Clinical Information, n.d.). SSC (Simons Simplex Collection) Database was created in 2011, with genetic samples and medical and behavioral data from 2,600 simplex families (SFARI | Simons Simplex Collection, 2011). They also conducted a followup project in 2015 and have follow-up data from 440 families. This database collects information through 29 different phenotypic and medical questionnaires, supplementary demographic data, and genetic data. The list of tests can be found in Supplementary II.B. SPARK (Simons Foundation Powering Autism Research for Knowledge) is "an autism research initiative that aims to recruit, engage and retain a community of 50,000 individuals with autism and their family members living in the U.S. "(SFARI $\mid$ SPARK, 2018). This data gathering started in 2015 and now is extended to about 90.000 ASD cases (SFARI $\mid$ SPARK Update, 2020). Used questionnaires are ADOS, DCDQ, VABS-III, SCQ, RBS-R, CBCL. These databases are available after prior authorization. 
In analyzing autism phenotypes, these or other broad databases provide an opportunity to examine the heterogeneity of ASD from several perspectives. Some researchers create and use their databases despite the comprehensive databases, which are sometimes too small to reach high statistical power. That fact might limit the conclusions drawn from the results (Norcia et al., 2021) (Macari et al., 2021).

Problems also can quickly occur with data gathering. Even if the sample size is large enough, if the chosen individuals are not forming a representative sample, the conclusions that can be driven from these analyses are limited. We found only a few studies that stated that the sample is representative (McCormick et al., 2020) (Maenner et al., 2020) (Randall et al., 2016)

\section{Questionnaires}

The results of a study can better cover the heterogeneity of ASD when it uses data from several questionnaires (e.g., ADOS, ADI-R, SCQ, SRS) and a broad database (e.g., SPARK or SFARI).

The most frequently used behavioral questionnaires are $A D O S, A D I-R, S R S$. Other behavioral and cognitive tests like $C B C L, S C Q, V A B S, M E S L$, and $W I S C$ are also used to supplement the former tests. There is some overlap, but there are also several differences between these questionnaires. Some of these instruments use developmental history (e.g., ADI-R), and others record current symptoms (e.g., ADOS). Some are conducted by examining the child by a trained rater (e.g., ADOS); others are based on parental responses (e.g., SRS). Given these differences and the opportunities, all tests have their limitations, which is why researchers use several questionnaires. The following section summarizes the features of the three most used 
behavioral tests: ADI-R, ADOS, and SRS, giving a brief description of each questionnaire, with advantages and disadvantages from a research point of view.

The Autism Diagnostic Interview-Revised (ADI-R) is a 93-question test system consisting of three parts (Lord et al., 1994) ((ADI $\left.{ }^{T M}-R\right)$ Autism Diagnostic Interview-Revised, n.d.). ADIR-A tests social, emotional, and reciprocal behavior, ADI-R-B tests communication, and verbal skills, and ADI-R-C tests repetitive, unusual, single-focus movements, speech, and behavior. Regarding the scoring, 0-3 points can be given per test question; higher points indicate a more severe change on an ordinal scale. The questionnaire also describes the child's medical history, milestones, and current state of development of speech and behavior (What Is ADI-R and How Does It Work? 2017). ADI-R does not directly involve the child in the study; it is an interview conducted by a qualified researcher or psychologist with the parent. The limitation of this questionnaire is that there are a relatively large number of questions related to verbal skills, which cannot be answered with appreciation in children who are too young or have problems with verbal development. This test system focuses primarily on the child's developmental history, not just the current condition, which can be an advantage, but indirect information (not the child being examined by the tester but relying on the caregiver's responses) can sometimes be misleading.

The Social Responsiveness Scale (SRS) is a 65-item questionnaire designed to assess social behavior problems associated with autism ((SRS $S^{T M}$-2) Social Responsiveness Scale, Second Edition, n.d.). The questions in the SRS test are divided into five subgroups: Social Awareness, Social Cognition, Social Communication, Social Motivation, Autistic Mannerisms. Regarding scoring, 1-4 points can be given per test question (1-not true, 2sometimes true, 3-often true, 4-almost always true). To always have a higher score to indicate a more severe deviation, in some cases, the original score must be reversed (replacing "1" with " 4 ", and "2" with " 3 "), which is indicated by the letter " $R$ " next to the name of the test 
question. The SRS does not directly involve the child in the study; the parent completes the questionnaire without the assistance of a trained examiner. That has some advantages, as the parent has the most information about the child and has limitations, as this assessment is subjective.

The Autism Diagnostic Observation Scheme (ADOS) test system is a unified and systematic method for diagnosing ASD (ADOS $S^{T M}$ - Autism Diagnostic Observation Schedule ${ }^{T M}$, n.d.) (Carr, 2013). The test system consists of four different modules, designed according to individuals of different ages and communication abilities. The improved version, ADOS 2, also has a separate "toddler module" for the youngest patients (Autism Diagnostic Observation Schedule, 2nd Edition (ADOS-) | Center for Autism Research, n.d.). The primary aspect of module selection is the subject's verbal communication level. Each module is divided into five parts, with appropriate questions regarding communication (part a.), reciprocal social interaction (part b.), fantasy (part c.), and rare, non-specific problems, like restrictive, repetitive behaviors (part d.) and anxiety-related problems (part e.)

Individual behaviors are assessed on a scale of 0 to 2 or 0 to 3 for 28-29 different questions (traits) in each module, with a higher score indicating an increased deviation from the behavior of the typically developing and behaving individuals.

The advantages of using data from ADOS tests for research are that it is objective and well documented. A trained examiner performs it, and it is based on the direct observation of the individual under predetermined, reproducible conditions. The questionnaire results are recorded in numerical codes (How Is ADOS (Autism Diagnostic Observation Schedule) Used to Identify ASD? n.d.).

As it is an assessment tool developed initially for diagnostics, limitations also occur if used for research. In each module, the characteristics of the individual are measured through age- 
and verbal-level-appropriate situations, so the elements of each module only partially match. Analyzes for research are somewhat complicated as elements of the same content may have a different serial number or, in some cases, a different wording. (Examples in Table 1)

Table 1

Examples of different ADOS features in the four modules(ADOS ${ }^{\mathrm{TM}}$ - Autism Diagnostic Observation Schedule ${ }^{\mathrm{TM}}$, n.d.)

\begin{tabular}{|l|l|l|l|}
\hline Module 1 & Module 2 & Module 3 & Module 4 \\
\hline A4_immediate echolalia & A4_immediate echolalia & A3_immediate echolalia & A3_immediate echolalia \\
\hline A5_stereotyped words & A5_stereotyped words & A4_stereotyped words & A4_stereotyped words \\
\hline B12_social overture & B8_quality overture & B7_quality overture & B9_social overture \\
\hline
\end{tabular}

Future research may consider that some ADOS traits, which are initially in the communication part (a.), could belong to the questionnaire's social interaction part (b.). Others might better fit in the part that records rare, non-specific (d., e.) behaviors, as in the improved ADOS-2 test system ((ADOS®-2) Autism Diagnostic Observation ScheduleTM, Second Edition, n.d., p. 2).

Analyzers should consider that values for given characteristics could be $0-3$ or $0-2$ depending on the item in question. For research analysis, it should also be noted that the 0-2 and 0-3 rating scales cannot be interpreted as interval scales, only as ordinal scales, as the numbers symbolize the increasing severity of the symptom. Still, the extent of change between two values cannot be quantified. Therefore, the incidence of each trait-value pair and their cooccurrences can be compared and analyzed safely, but care is needed for other types of evaluations.

Another limitation in using data from ADOS questionnaires is the problem of "unsigned evaluation": the assessment only expresses the severity of the symptom but does not indicate whether we are facing a negative (lack of typical behavior) or positive (appearance of atypical symptom) change. This problem could be solved by using values with negative or positive signs during the test. In that case, the severity of the condition is indicated by the absolute 
value of the score and its character by its sign. If used for research, the data should not be evaluated using a method based on scale relations (e.g., averaging to 0 ).

If an existing database is analyzed, the video of the survey may be a solution to distinguish between the two options. By analyzing the video, information can be obtained whether there is a negative or positive deviation in each case.

\section{Analysis tools}

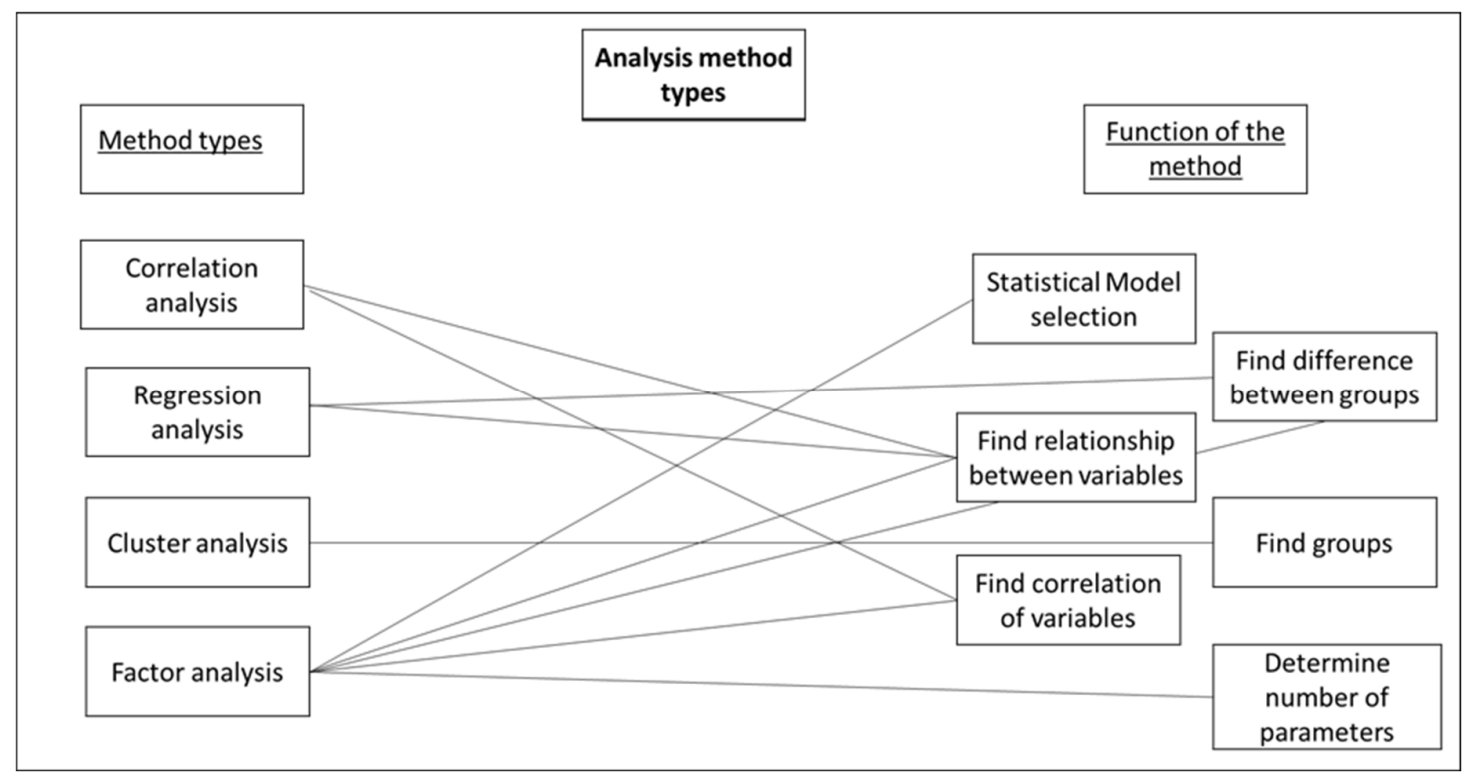

Figure 8

Analysis methods

The type of analysis tools used in research depends on the goal of the study

There are different analysis tools commonly used in analyzing autistic phenotypes. The method of approach, the type and size of the database, the number, and the nature of the parameters selected all influence which methods should be used. (See Supplementary I.B. "methods in papers" for detailed information) 
The goal of the analyses could be one or more of the following: 1) to determine the number of the parameters to be used to analyze the data, 2) to select the appropriate statistical model, 3) to determine the ideal number of groups within the sample and to define the groups according to the difference in the values of one or more parameters, 4) classification of elements into the groups obtained previously, and 5) to search for the relationship between the chosen variables or correlation of the selected parameters. (Figure 10)

When using a supervised approach, the first step is selecting a grouping factor (X). Groups are then defined by the different values of this grouping factor. The second step is to choose one or more dependent variables (Y). After that, the following questions should be answered: Are the groups formed based on the grouping factor $(\mathrm{X})$ significantly different in terms of the dependent variable $(\mathrm{Y})$ ? Are the groups more different from each other than the degree of heterogeneity experienced within the group (in other words: is within-group variance less than the difference in mean values of the different groups)? To answer these questions, the researcher should choose the appropriate hypothesis testing methods, correlation-, latent variable-, factor- or cluster-analyses.

Exploratory factor analyses can be used to determine the ideal number of parameters that should be examined (Exploratory Factor Analysis | Columbia Public Health, n.d.). To select the appropriate statistical model and variables, we found the following methods in the reviewed articles: Bayesian Information Criterion (BIC) helps choose the most important descriptive variables (Stephanie, 2018). Bootstrap likelihood ratio test (BLRT) (Tein et al., 2013) or Lo-Mende-Rubin likelihood ratio test (LMR-LRT) can be used to compare two statistical models, indicating which one fits better to the observed data. Classifiers, such as Random Forest Algorithm (Classification Algorithms - Random Forest, n.d.) or Gradient Boosting Classifier, can also be used for model selection. 
When using an unsupervised approach, the first step is to determine the ideal number of groups, which can be done using Factor-analysis, Latent profile analysis (Spurk et al., 2020), or Multivariate latent class growth analyses. Cluster analysis methods (Cluster Analysis | Statistics | Britannica, n.d.), such as Hierarchical or Model-based cluster analyses, can also be used to organize the data into separate groups, even when using several variables. The analyses themselves define the grouping factors and the groups. These methods also show the difference between the groups because they divide the data into classes that are significantly different from each other.

Regression analyses show the relationship between continuous variables (Regression Analysis, n.d.). Such methods can also be utilized to recover differences between the groups regarding this information.

To determine the difference between two or more predefined groups, Fisher's ExactTest and, Chi-square test can be applied with nominal data (Chi-Square Statistic, n.d.), and t-probes or $\boldsymbol{A N O V A}$ (analysis of variance), if the data ranges on an interval scale (ANOVA Test, n.d.). The relationship and correlation of the variables should be analyzed with Pearson correlation or Linear regression analysis for continuous data and Spearman correlation analysis for ordinal data (Linear Regression, n.d.). Factor analysis can also be used to study the relationship and correlation of the variables. The type of method depends on the type of data analyzed (see details in Supplemental I.C.). The possibilities and limitations of the tools used are mainly determined by the nature of the chosen parameters (nominal/ordinal/quantitative) and the type of question (finding similarities or differences).

When choosing the proper analysis tool, the sample size should also be considered. Some tests, such as Fisher's Exact Test, can be used with small sample sizes, but most tests, for

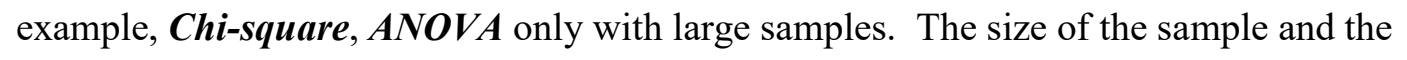
number of parameters that can be analyzed are also correlated. The bigger the sample, the 
more variables can be used. For example, the sample size must be at least five times the number of parameters tested for factor analyses.

When designing research, it is essential to choose the appropriate analysis tools regarding not only the goal of the study but also the possibilities and limitations that originate from the type and size of the database and the type and number of parameters to be examined.

\section{Aspects in the formation of the subgroups}

As for case-control- and heterogeneity models, we are researching only the phenotypic aspects of autism subtyping in this review. When designing a study on subtypes of ASD, there are two possible ways to start. Some researchers designate a leading aspect and organize data to find subgroups according to the selected criterion. In these cases, called the supervised approach, the research "deals with a priori knowledge about a topic (i.e., known labels), and then seeks to derive a model to predict best that known information" (Lombardo et al., 2019). They usually use this approach in case-control studies and subgrouping from a specific aspect, such as sensory processing problems or motoric disabilities linked to autistic traits (Dziuk et al., 2007) (Norcia et al., 2021).

A different approach is to start from a computational angle instead of pre-selecting the aspects. In this case, "the learning occurs without any expert a priori knowledge, and justification solely relies on statistical distinctions embedded in the data itself' (Lombardo et al., 2019). This is called the unsupervised or data-driven approach. For example, it is used when hierarchical clusters are made (Obafemi-Ajayi et al., 2015) or in unsupervised machine learning (Stevens et al., 2019). In this way, the subgroups that emerge from the study are usually determined by more than one aspect, for example, different social and RRB severity subtypes (Klopper et al., 2017), or subtypes formed concerning developmental, motor, language, and restrictive-repetitive traits (Wiggins et al., 2017). 
As outlined, different subgroups can be obtained in the examined sample due to autism subtyping. The Encyclopedia of ASD states that subtypes or subgroups ,, can be defined as groups of persons with autism spectrum disorders who are more similar to each other than to the larger group of individuals who fall under the broad diagnostic category of ASD" (Hus et al., 2013). We think that the determination of similarity largely depends on how many parameters are considered and which ones are selected from the available data. Results may also depend on the methods used to analyze the data; methods may perform better or worse subject to the statistics of the data. Based on the pre-selection of groups, traits, or broader data analysis, one may obtain different results, and conclusions should be considered with care. Discrepancies may result from the wild variation of the causes of autism or the potential causes of significant statistical discrepancies in different cohorts. An intriguing example is the minicolumnar hypothesis of autism, where results contradict each other except the conclusion that microcircuits are altered in autism. (Casanova et al., 2002) (McKavanagh et al., 2015) (Park et al., 2021)

\section{Subgroups in autism}

The most common subgroups reported in the selected papers are described below.

Overall severity subgroups usually refer to social severity, with individuals having different problems with social motivation and awareness, social cognition, and communication. For example, in a recent study, they found five profiles: 1) socially adaptive, 2) mild, 3) moderate A: social responsiveness problems, 4) moderate B: problems with expressing social communication, 5) severe: worst in all aspects (Uljarević et al., 2020). This study found no difference in chronological age or gender among the subgroups they found in their research. 
Researchers are measuring deficits in IQ and component-based cognition when searching for cognitive subgroups. In a study from 2014 on Nonverbal and Verbal Cognitive Discrepancy, they found that the ratio of verbal and nonverbal IQ was influenced by age and gender (Ankenman et al., 2014). Another study from 2018 (Feczko et al., 2018) found three subgroups in a relatively small sample $(\mathrm{N}=47)$ with the characteristics of 1$)$ altered visual processing, 2) altered attention mechanisms, 3) both. They found no association with age and gender or autism symptom severity that could subgroup the individuals in this study. The difference may originate from the differences in the questions asked, the small number of subjects, and the selected cohorts' differences.

In forming sensory subgroups, the researchers examine problems of visual, taste, and auditory perceptions (Lane et al., 2011) (Tomchek et al., 2018). The results are heterogeneous; a typical classification is forming four subgroups: (a) sensory adaptive; (b) taste smell sensitive; (c) postural inattentive; and (d) generalized sensory difference (Lane et al., 2014). Other researchers recommend considering sensory features as dimensional variables and "adopting a comprehensive, multidimensional and multimodal approach to the measurement of sensory features" (Uljarević et al., 2017).

Motoric subgroups can be formed along with different aspects. Researchers could examine deficits in motor skills, voice and word formation, and planning/reprogramming movements (Dziuk et al., 2007) (Dowd et al., 2012).

Restrictive and repetitive behaviors usually contain many motoric elements, so in a way, these subtypes can also be included into this group (Zheng et al., 2019). From another point of view, RRB often has connections to anxiety (Russell et al., 2019).

Separating subtypes or subgroups in the studied group of autistic individuals can help make a more accurate diagnosis and develop appropriate therapy. For this reason, the identification of 
subtypes and the classification of individuals into these subgroups can be essential for early diagnosis and treatment planning.

\section{Conclusions}

The phenomenon of autism, its development, and the causes of its heterogeneous symptoms raise many questions. The problem is that in the absence of a sufficiently accurate diagnosis, the design of effective treatment is questionable. An important line of research to aid diagnosis is clustering individuals with autism into smaller subgroups. These subgroups should be more homogeneous regarding their leading symptoms and developmental trajectories. This may pave the way for a better understanding and thus more successful, individualized treatments.

The wide variety of autistic features, such as the severity groups, cognitive subgroups, sensory subgroups, motoric subgroups, and the potential comorbidities not treated here, and the rareness of some of the characteristics form significant challenges concerning the database, questionnaires, tests and methods applied. Due to the many dimensions of autistic traits and the combinations of potential causes that can lead to impairments in social communication, it is easy to underestimate the number of individuals that can form a representative sample. Therefore, sometimes significant looking outcomes may be misleading.

Our article summarized the different aspects, concepts, possibilities, and limitations of autistic phenotype analysis by systematization and synthesis of information gained from carefully selected research papers. This work might bring a conceptual overview of this heterogeneous topic. 


\section{Reference list}

$\left(A D I^{T M}-R\right)$ Autism Diagnostic Interview-Revised. (n.d.). Retrieved 3 December 2021, from https://www.wpspublish.com/adi-r-autism-diagnostic-interviewrevised

$\left(\right.$ ADOS $\left.^{\circledR}-2\right)$ Autism Diagnostic Observation Schedule ${ }^{T M}$, Second Edition. (n.d.). Retrieved 3 December 2021, from https://www.wpspublish.com/ados-2-autism-diagnostic-observation-schedulesecond-edition

ADOS $^{T M}$-Autism Diagnostic Observation Schedule ${ }^{T M}$. (n.d.). Retrieved 3 December 2021, from https://eprovide.mapi-trust.org/instruments/autism-diagnostic-observation-schedule

AGRE Program Description. (n.d.). Autism Speaks. Retrieved 2 December 2021, from https://www.autismspeaks.org/agre-program-description

American Psychiatric Association, \& American Psychiatric Association (Eds.). (2013). Diagnostic and statistical manual of mental disorders: DSM-5 (5th ed). American Psychiatric Association.

Anderson, G. M., Montazeri, F., \& de Bildt, A. (2015). Network Approach to Autistic Traits: Group and Subgroup Analyses of ADOS Item Scores. Journal of Autism and Developmental Disorders, 45(10), 3115-3132. https://doi.org/10.1007/s10803-015-2537-z

Ankenman, K., Elgin, J., Sullivan, K., Vincent, L., \& Bernier, R. (2014). Nonverbal and Verbal Cognitive Discrepancy Profiles in Autism Spectrum Disorders: Influence of Age and Gender. American Journal on Intellectual and Developmental Disabilities, 119(1), 84-99.

https://doi.org/10.1352/1944-7558-119.1.84

ANOVA Test: Definition, Types, Examples, SPSS. (n.d.). Statistics How To. Retrieved 7 December 2021, from https://www.statisticshowto.com/probability-and-statistics/hypothesis-testing/anova/ Autism Diagnostic Observation Schedule, 2nd Edition (ADOS-) / Center for Autism Research. (n.d.). Retrieved 3 December 2021, from https://www.carautismroadmap.org/autism-diagnosticobservation-schedule-ados/ 
Baadel, S., Thabtah, F., \& Lu, J. (2020). A clustering approach for autistic trait classification. Informatics for Health and Social Care, 45(3), 309-326. https://doi.org/10.1080/17538157.2019.1687482

Becht, A. I., Nelemans, S. A., Branje, S. J. T., Vollebergh, W. A. M., Koot, H. M., Denissen, J. J. A., \& Meeus, W. H. J. (2016). The quest for identity in adolescence: Heterogeneity in daily identity formation and psychosocial adjustment across 5 years. Developmental Psychology, 52(12), 2010-2021. https://doi.org/10.1037/dev0000245

C Yuen, R. K., Merico, D., Bookman, M., L Howe, J., Thiruvahindrapuram, B., Patel, R. V., Whitney, J., Deflaux, N., Bingham, J., Wang, Z., Pellecchia, G., Buchanan, J. A., Walker, S., Marshall, C. R., Uddin, M., Zarrei, M., Deneault, E., D’Abate, L., Chan, A. J. S., ... Scherer, S. W. (2017). Whole genome sequencing resource identifies 18 new candidate genes for autism spectrum disorder. Nature Neuroscience, 20(4), 602-611. https://doi.org/10.1038/nn.4524

Carr, T. (2013). Autism Diagnostic Observation Schedule. In F. R. Volkmar (Ed.), Encyclopedia of Autism Spectrum Disorders (pp. 349-356). Springer. https://doi.org/10.1007/978-1-44191698-3_896

Casanova, M. F., Buxhoeveden, D. P., Switala, A. E., \& Roy, E. (2002). Minicolumnar pathology in autism. Neurology, 58(3), 428-432. https://doi.org/10.1212/WNL.58.3.428

Chi-Square Statistic: How to Calculate It / Distribution. (n.d.). Statistics How To. Retrieved 7 December 2021, from https://www.statisticshowto.com/probability-and-statistics/chisquare/

Classification Algorithms-Random Forest. (n.d.). Retrieved 3 December 2021, from https://www.tutorialspoint.com/machine_learning_with_python/classification_algorithms_r andom_forest.htm

Cluster analysis / statistics / Britannica. (n.d.). Retrieved 3 December 2021, from https://www.britannica.com/topic/cluster-analysis 
Constantino, J. N., \& Charman, T. (2016). Diagnosis of autism spectrum disorder: Reconciling the syndrome, its diverse origins, and variation in expression. The Lancet Neurology, 15(3), 279291. https://doi.org/10.1016/S1474-4422(15)00151-9

Dowd, A. M., McGinley, J. L., Taffe, J. R., \& Rinehart, N. J. (2012). Do Planning and Visual Integration Difficulties Underpin Motor Dysfunction in Autism? A Kinematic Study of Young Children with Autism. Journal of Autism and Developmental Disorders, 42(8), 1539-1548. https://doi.org/10.1007/s10803-011-1385-8

Dziuk, M. A., Gidley Larson, J. C., Apostu, A., Mahone, E. M., Denckla, M. B., \& Mostofsky, S. H. (2007). Dyspraxia in autism: Association with motor, social, and communicative deficits. Developmental Medicine and Child Neurology, 49(10), 734-739. https://doi.org/10.1111/j.1469-8749.2007.00734.x

Exploratory Factor Analysis / Columbia Public Health. (n.d.). Retrieved 3 December 2021, from https://www.publichealth.columbia.edu/research/population-health-methods/exploratoryfactor-analysis

Feczko, E., Balba, N. M., Miranda-Dominguez, O., Cordova, M., Karalunas, S. L., Irwin, L., Demeter, D. V., Hill, A. P., Langhorst, B. H., Grieser Painter, J., Van Santen, J., Fombonne, E. J., Nigg, J. T., \& Fair, D. A. (2018). Subtyping cognitive profiles in Autism Spectrum Disorder using a Functional Random Forest algorithm. Neurolmage, 172, 674-688. https://doi.org/10.1016/j.neuroimage.2017.12.044

Feczko, E., Miranda-Dominguez, O., Marr, M., Graham, A. M., Nigg, J. T., \& Fair, D. A. (2019). The Heterogeneity Problem: Approaches to Identify Psychiatric Subtypes. Trends in Cognitive Sciences, 23(7), 584-601. https://doi.org/10.1016/j.tics.2019.03.009

Fóthi, Á., Soorya, L., \& Lőrincz, A. (2020). The Autism Palette: Combinations of Impairments Explain the Heterogeneity in ASD. Frontiers in Psychiatry, 11, 503462. https://doi.org/10.3389/fpsyt.2020.503462 
Haslbeck, J. M. B., \& Fried, E. I. (2017). How predictable are symptoms in psychopathological networks? A reanalysis of 18 published datasets. Psychological Medicine, 47(16), 2767-2776. https://doi.org/10.1017/S0033291717001258

Hollander, E., Cartwright, C., Wong, C. M., DeCaria, C. M., DelGiudice-Asch, G., Buchsbaum, M. S., \& Aronowitz, B. R. (1998). A Dimensional Approach to the Autism Spectrum. CNS Spectrums, 3(3), 22-39. https://doi.org/10.1017/S1092852900005678

How is ADOS (Autism Diagnostic Observation Schedule) Used to Identify ASD? (n.d.). Retrieved 3 December 2021, from https://www.appliedbehavioranalysisedu.org/how-is-ados-autismdiagnostic-observation-schedule-used-to-identify-asd/

Hus, V., Kim, S. H. S., \& Lord, C. (2013). Subtyping Autism. In F. R. Volkmar (Ed.), Encyclopedia of Autism Spectrum Disorders (pp. 3027-3036). Springer. https://doi.org/10.1007/978-1-44191698-3_918

Katuwal, G. J., Baum, S. A., Cahill, N. D., \& Michael, A. M. (2016). Divide and Conquer: Sub-Grouping of ASD Improves ASD Detection Based on Brain Morphometry. PLOS ONE, 11(4), e0153331. https://doi.org/10.1371/journal.pone.0153331

Kim, H., Keifer, C., Rodriguez-Seijas, C., Eaton, N., Lerner, M., \& Gadow, K. (2018). Quantifying the Optimal Structure of the Autism Phenotype: A Comprehensive Comparison of Dimensional, Categorical, and Hybrid Models. Journal of the American Academy of Child \& Adolescent Psychiatry, 58. https://doi.org/10.1016/j.jaac.2018.09.431

Klopper, F., Testa, R., Pantelis, C., \& Skafidas, S. (2017). A cluster analysis exploration of autism spectrum disorder subgroups in children without intellectual disability. Research in Autism Spectrum Disorders, 36, 66-78. https://doi.org/10.1016/j.rasd.2017.01.006

Lane, A. E., Dennis, S. J., \& Geraghty, M. E. (2011). Brief Report: Further Evidence of Sensory Subtypes in Autism. Journal of Autism and Developmental Disorders, 41(6), 826-831. https://doi.org/10.1007/s10803-010-1103-y 
Lane, A. E., Molloy, C. A., \& Bishop, S. L. (2014). Classification of Children With Autism Spectrum Disorder by Sensory Subtype: A Case for Sensory-Based Phenotypes. Autism Research, 7(3), 322-333. https://doi.org/10.1002/aur.1368

Linear Regression: Simple Steps, Video. Find Equation, Coefficient, Slope. (n.d.). Statistics How To. Retrieved 7 December 2021, from https://www.statisticshowto.com/probability-andstatistics/regression-analysis/find-a-linear-regression-equation/

Lombardo, M. V., Lai, M.-C., \& Baron-Cohen, S. (2019). Big data approaches to decomposing heterogeneity across the autism spectrum. Molecular Psychiatry, 24(10), 1435-1450. https://doi.org/10.1038/s41380-018-0321-0

Lord, C., Rutter, M., \& Le Couteur, A. (1994). Autism Diagnostic Interview-Revised: A revised version of a diagnostic interview for caregivers of individuals with possible pervasive developmental disorders. Journal of Autism and Developmental Disorders, 24(5), 659-685. https://doi.org/10.1007/BF02172145

Macari, S. L., Vernetti, A., \& Chawarska, K. (2021). Attend Less, Fear More: Elevated Distress to Social Threat in Toddlers With Autism Spectrum Disorder. Autism Research, 14(5), 1025-1036. https://doi.org/10.1002/aur.2448

Maenner, M. J., Shaw, K. A., Baio, J., EdS1, Washington, A., Patrick, M., DiRienzo, M., Christensen, D. L., Wiggins, L. D., Pettygrove, S., Andrews, J. G., Lopez, M., Hudson, A., Baroud, T., Schwenk, Y., White, T., Rosenberg, C. R., Lee, L.-C., Harrington, R. A., ... Dietz, P. M. (2020). Prevalence of Autism Spectrum Disorder Among Children Aged 8 Years-Autism and Developmental Disabilities Monitoring Network, 11 Sites, United States, 2016. MMWR. Surveillance Summaries, 69(4), 1-12. https://doi.org/10.15585/mmwr.ss6904a1

McCormick, C. E. B., Kavanaugh, B. C., Sipsock, D., Righi, G., Oberman, L. M., Luca, D. M. D., Uzun, E. D. G., Best, C. R., Jerskey, B. A., Quinn, J. G., Jewel, S. B., Wu, P.-C., McLean, R. L., Levine, T. P., Tokadjian, H., Perkins, K. A., Clarke, E. B., Dunn, B., Gerber, A. H., ... Morrow, E. M. (2020). Autism Heterogeneity in a Densely Sampled U.S. Population: Results From the First 1,000 
Participants in the RI-CART Study. Autism Research, 13(3), 474-488.

https://doi.org/10.1002/aur.2261

McKavanagh, R., Buckley, E., \& Chance, S. A. (2015). Wider minicolumns in autism: A neural basis for altered processing? Brain, 138(7), 2034-2045. https://doi.org/10.1093/brain/awv110

MSSNG. (n.d.). Retrieved 2 December 2021, from https://research.mss.ng/

Norcia, A. M., Lee, A., Meredith, W. J., Kohler, P. J., Pei, F., Ghassan, S. A., Libove, R. A., Phillips, J. M., \& Hardan, A. Y. (2021). A case-control study of visual, auditory and audio-visual sensory interactions in children with autism spectrum disorder. Journal of Vision, 21(4), 5.

https://doi.org/10.1167/jov.21.4.5

Obafemi-Ajayi, T., Lam, D., Takahashi, T. N., Kanne, S., \& Wunsch, D. (2015). Sorting the phenotypic heterogeneity of autism spectrum disorders: A hierarchical clustering model. 2015 IEEE Conference on Computational Intelligence in Bioinformatics and Computational Biology (CIBCB), 1-7. https://doi.org/10.1109/CIBCB.2015.7300337

Page, M. J., McKenzie, J. E., Bossuyt, P. M., Boutron, I., Hoffmann, T. C., Mulrow, C. D., Shamseer, L., Tetzlaff, J. M., Akl, E. A., Brennan, S. E., Chou, R., Glanville, J., Grimshaw, J. M., Hróbjartsson, A., Lalu, M. M., Li, T., Loder, E. W., Mayo-Wilson, E., McDonald, S., ... Moher, D. (2021). The PRISMA 2020 statement: An updated guideline for reporting systematic reviews. BMJ, 372, n71. https://doi.org/10.1136/bmj.n71

Park, B., Hong, S.-J., Valk, S. L., Paquola, C., Benkarim, O., Bethlehem, R. A. I., Di Martino, A., Milham, M. P., Gozzi, A., Yeo, B. T. T., Smallwood, J., \& Bernhardt, B. C. (2021). Differences in subcortico-cortical interactions identified from connectome and microcircuit models in autism. Nature Communications, 12(1), 2225. https://doi.org/10.1038/s41467-021-21732-0

Randall, M., Sciberras, E., Brignell, A., Ihsen, E., Efron, D., Dissanayake, C., \& Williams, K. (2016). Autism spectrum disorder: Presentation and prevalence in a nationally representative Australian sample. The Australian and New Zealand Journal of Psychiatry, 50(3), 243-253. https://doi.org/10.1177/0004867415595287 
Regression Analysis: Step by Step Articles, Videos, Simple Definitions. (n.d.). Statistics How To.

Retrieved 7 December 2021, from https://www.statisticshowto.com/probability-andstatistics/regression-analysis/

Russell, K. M., Frost, K. M., \& Ingersoll, B. (2019). The relationship between subtypes of repetitive behaviors and anxiety in children with autism spectrum disorder. Research in Autism Spectrum Disorders, 62, 48-54. https://doi.org/10.1016/j.rasd.2019.03.006

SFARI / Simons Simplex Collection. (2011, August 19). SFARI. https://www.sfari.org/resource/simonssimplex-collection/

SFARI / SPARK. (2018, February 7). SFARI. https://www.sfari.org/resource/spark/

SFARI | SPARK Demographic and Clinical Information. (n.d.). SFARI. Retrieved 2 December 2021, from https://www.sfari.org/spark-demographic-and-clinical-information/

SFARI / SPARK Phenotypic Measures. (n.d.). SFARI. Retrieved 2 December 2021, from https://www.sfari.org/spark-phenotypic-measures/

SFARI / SPARK update: New phenotypic data now available. (2020, May 18). SFARI. https://www.sfari.org/2020/05/18/spark-update-new-phenotypic-data-now-available/

Spurk, D., Hirschi, A., Wang, M., Valero, D., \& Kauffeld, S. (2020). Latent profile analysis: A review and "how to" guide of its application within vocational behavior research. Journal of Vocational Behavior, 120, 103445. https://doi.org/10.1016/j.jvb.2020.103445

(SRS ${ }^{T M}$-2) Social Responsiveness Scale, Second Edition. (n.d.). Retrieved 3 December 2021, from https://www.wpspublish.com/srs-2-social-responsiveness-scale-second-edition Stephanie. (2018, March 10). Bayesian Information Criterion (BIC) / Schwarz Criterion. Statistics How To. https://www.statisticshowto.com/bayesian-information-criterion/

Stevens, E., Dixon, D. R., Novack, M. N., Granpeesheh, D., Smith, T., \& Linstead, E. (2019). Identification and analysis of behavioral phenotypes in autism spectrum disorder via unsupervised machine learning. International Journal of Medical Informatics, 129, 29-36. https://doi.org/10.1016/j.ijmedinf.2019.05.006 
Tang, S., Sun, N., Floris, D. L., Zhang, X., Di Martino, A., \& Yeo, B. T. T. (2020). Reconciling Dimensional and Categorical Models of Autism Heterogeneity: A Brain Connectomics and Behavioral Study. Biological Psychiatry, 87(12), 1071-1082. https://doi.org/10.1016/j.biopsych.2019.11.009

Tein, J.-Y., Coxe, S., \& Cham, H. (2013). Statistical Power to Detect the Correct Number of Classes in Latent Profile Analysis. Structural Equation Modeling: A Multidisciplinary Journal, 20(4), 640657. https://doi.org/10.1080/10705511.2013.824781

Tomchek, S. D., Little, L. M., Myers, J., \& Dunn, W. (2018). Sensory Subtypes in Preschool Aged Children with Autism Spectrum Disorder. Journal of Autism and Developmental Disorders, 48(6), 2139-2147. https://doi.org/10.1007/s10803-018-3468-2

Uljarević, M., Baranek, G., Vivanti, G., Hedley, D., Hudry, K., \& Lane, A. (2017). Heterogeneity of sensory features in autism spectrum disorder: Challenges and perspectives for future research. Autism Research, 10(5), 703-710. https://doi.org/10.1002/aur.1747

Uljarević, M., Phillips, J. M., Schuck, R. K., Schapp, S., Solomon, E. M., Salzman, E., Allerhand, L., Libove, R. A., Frazier, T. W., \& Hardan, A. Y. (2020). Exploring Social Subtypes in Autism Spectrum Disorder: A Preliminary Study. Autism Research, 13(8), 1335-1342. https://doi.org/10.1002/aur.2294

What is ADI-R and How Does it Work? (2017, September 7). https://www.appliedbehavioranalysisedu.org/what-is-adi-r-and-how-does-it-work/ What is the GRADE-CERQual approach: Grade-cerqual. (2019, October 2). https://www.cerqual.org/what-is-the-grade-cerqual-approach2/

Wiggins, L. D., Tian, L. H., Levy, S. E., Rice, C., Lee, L.-C., Schieve, L., Pandey, J., Daniels, J., Blaskey, L., Hepburn, S., Landa, R., Edmondson-Pretzel, R., \& Thompson, W. (2017). Homogeneous Subgroups of Young Children with Autism Improve Phenotypic Characterization in the Study to Explore Early Development. Journal of Autism and Developmental Disorders, 47(11), 36343645. https://doi.org/10.1007/s10803-017-3280-4 
WING, L. (1996). The Autistics Spectrum: Aguide for parents and professionals. The Autistics Spectrum : A Guide for Parents and Professionals. https://ci.nii.ac.jp/naid/20001667036/

Zheng, L., Grove, R., \& Eapen, V. (2019). Spectrum or subtypes? A latent profile analysis of restricted and repetitive behaviours in autism. Research in Autism Spectrum Disorders, 57, 46-54. https://doi.org/10.1016/j.rasd.2018.10.003 